\title{
How to Develop Skills of Designers and be Ready for Business?
}

\author{
Tomas Chochole \\ Ladislav Sutnar Faculty of Design and Art, University of West Bohemia, Pilsen, Czech Republic.
}

How to cite this paper: Tomas Chochole. (2021) How to Develop Skills of Designers and be Ready for Business? Journal of Humanities, Arts and Social Science, 5(1), 35-39.

DOI: 10.26855/jhass.2021.01.005

Received: December 31, 2020

Accepted: January 25, 2021

Published: February 6, 2021

*Corresponding author: Tomas Chochol, Ladislav Sutnar Faculty of Design and Art, University of West Bohemia, Pilsen, Czech Republic.

Email: chochole@fdu.zcu.cz

\begin{abstract}
The year 2020 significantly expanded our communication possibilities through a global pandemic. We have become people online. In the world of product design, where the final work depends on the cooperation of several different professions, online communication has become key for teamwork and thus for a successful business. We all learned it on the fly as best we could. But if we know which soft skills, we need to strengthen to make our teamwork more effective and our results better, we do not have to go the way of trial and error. This study aimed to map key soft skills in product design students from three different universities and in a group of professional designers in the context of their successful work in a highly competitive market. It turned out that interdisciplinary work in the online mode is not completely simple for this target group, but at the same time the preferred competencies were named, that can be the basis for the opening of different educational courses at universities or at other professional institutions.
\end{abstract}

\section{Keywords}

Product Design, Interdisciplinarity, Teamwork, Soft Skills, Business

\section{Introduction}

Interdisciplinary cooperation is a phenomenon of recent years-especially in culture, art, and design. When you manage to work with people who specialize in other things and are good at what you are not, and you end up working on a joint project, it is one of the best things you can experience. Your natural boundaries will shift, and you know that you have become part of something that has exceeded your possibilities, and although it has not been easy, you have managed it together. I call it breaking down borders!

As a person in charge of projects and their implementation at the faculty of design and art, I create natural conditions for interdisciplinary cooperation of our students with students of other faculties of our university, but also with students of other faculties of foreign universities. Especially in the field of product and industrial design, it is quite easy. After all, if something new is to emerge, it is necessary to work in a team with students from other disciplines. The topics of sustainable development, ecology, modern technologies, and many others enter the work of designers that they can share with their colleagues from IT, engineering, mechatronics, healthcare, or agriculture. It is good to see that our students enjoy working together, although working in a team is not always easy and in addition to expertise, you also need soft competencies to work together effectively. Without physical contact only with audio-video conferencing platforms, interdisciplinary collaboration is even more complicated. So, I was interested in what kind of soft skills will product design students consider most important for successful cooperation during 2020 and how can it interact their strategy to be successful on the market with their products. This created international research, in which students from two Chinese universities also took part, because the situation with the COVID-19 pandemic was 
similar in our schools. In addition, I compared the results of the student research with the experience of professional product designers, who also had to work online within their teams. This paper is a summary of this research.

\section{Research Design}

The international survey concept was during year 2020 focused on group of 98 product design students from product design and at the same time professional product designers, who had experience with interdisciplinary teamwork. The research also sought to map a group of students' relationships to the market and their ability to be successful in the market with their products. Teamwork leads students to critical reflection and can prepare them very well for professional design practice (Findlay, 1997).

All respondents had to work online in recent months, and the aim of the study was to map their experience with the use of soft competencies in the distance form of interdisciplinary teamwork using digital technologies. Teamwork can very effectively support students' skills, in this case we do not have to talk only about hard skills, but especially about soft ones (Meizlish \& Anderson, 2018).

At a time when working on a project requires the knowledge and skills of various students (experts) in a team, we can consider such a team as "interdisciplinary" (Nancarrow, et al., 2015). These experts can be, for instance technical engineers, health professionals, farmers, economists, or sociologists. Their task is to work on common goals, outputs and very often on final products. The trend is that only few products can be created without knowledge of other disciplines that is usually too complicated for individuals. Therefore, a narrower, but more professional focus of individual team members is preferred.

"Soft competencies" help people in their self-development, that is, at work and in their personal lives. Most of them are, for example, competencies focused on communication, problem solving, time management or flexibility and thus indicate social interaction (Matteson, et al., 2016).

Hard skills, as opposed to soft ones, are specific to a particular job also in product design. The meaning of individual specific soft skills differs slightly, but the aim of this study was not to unify their definitions, because in general the meanings of these competencies are clear.

Interdisciplinary teamwork is best done when team members can meet, see each other, discuss problem-solving, and reach proposed goals together. However, the global pandemic situation in the year 2020 has forced many such teams to communicate online. The research was based on the principle of a case study with "journalist's investigative methods" (Walker, 2018) as an innovative approach with combination of quantitative and qualitative data analysis. The survey covered 86 participants from three different universities:

- Ladislav Sutnar Faculty of Design and Art, University of West Bohemia in Pilsen, Czech Republic,

- School of Design of East China Normal University, Shanghai China,

- College of Engineering and Design of Hunan Normal University in Changsha, China.

Results from student's research were compared also with 12 professional product designers from Czech Republic and China. The aim of research was to describe their preferred key skills and different reflection of their experience with design work at teams. It was also important to know if they needed a special type of skill to succeed in an extremely competitive market with their products.

\section{Case Study}

The reason why this research was aimed at students in the field of product design and at professional product designers was that these experts often come into direct interaction with other experts when developing new products and thus work in smaller or larger teams. In addition, the global pandemic situation has made contact teamwork impossible and has made it only in digital version. Research of the competencies needed for this type of collaboration by product designers was more or less logical.

All respondents were selected for the research based on predetermined parameters, such as that in 2020 they were involved in interdisciplinary teamwork, actively used digital communication platforms, worked on real projects, the output of which were at least functional prototypes.

The research methodology worked with a questionnaire, which included the Likert scale and data analysis. The results of the preferred competencies were analyzed and summarized. In addition, an online structured interview was conducted with some respondents, the aim of which was to map the designers' experience with interdisciplinary cooperation and to asked also about barriers defend to be successful on the market with their products. The results of the international survey were systematically recorded, as well as written records of interviews with respondents. 
The study thus captures the necessary competencies for digital communication of team members from the point of view of product designers and specifies which competencies need to be strengthened (also for business market) for both students and professional designers. Because the information is commonly available online and work communication has also been significantly digitized during this year. Students no longer must go to their school for know-how, because it is globally available (Han, et al., 2017).

Interdisciplinary projects are implemented in different ways at each of the mentioned universities and have different demands. For the needs of the case study was also determined the basic parameters of interdisciplinary projects:

- $\quad$ the project was solved online during year 2020,

- the solution of the project was possible only in cooperation of students from several different fields (technical engineering, agriculture, humanities, medical studies, economics, design, etc.),

- $\quad$ students of product design were involved in the project, work in teams together with students of other disciplines and had divided roles and responsibilities in their teams (team manager, developer, content guarantor, marketing strategist, etc.),

- the project assignment was formulated according to the practical needs of the university or in cooperation with business partners,

- students had the opportunity to continuously consult their work with the client of the topic online,

- $\quad$ several student teams worked simultaneously on one project assignment, and the contracting authority finally chose the best team solution,

- the output of the project was, for example, a model, a prototype, a functional sample with an accompanying description of key areas from each participating field.

Giving more responsibility to student teams, including their original authorship, often leads to unexpected, effective, and innovative results, and provides students with a unique learning experience (Stewart \& Clifford, 2018).

A basic research question was defined for the needs of the research:

- What kind of soft skills do you consider necessary for interdisciplinary teamwork with regard to business market success?

Investigative journalistic research methods were used for this research study, which combine an analytical and synthetic approach and consider additional interviews with some respondents. The used questionnaire contained besides 15 pre-defined soft competencies that were selected on the basis of data comparison based on international research of market needs in the field of product design.

The results of the international survey were systematically recorded, as well as written records of interviews with respondents.

The graph (Figure 1) shows the most preferred soft skills for students and professional designers-these include teamwork, effective communication, problem solving, critical thinking, or time management. Slight differences in preferences are influenced by the greater level of experience that professionals already have. It was very surprising to find that students also chose business skills as a part of the skills they consider important for their successful presence in the product market. In the case of professional designers, this skill was not preferred because they have already acquired a certain form of business skills and thus take them for granted. This is confirmed by experience in the field of design. "Designers need to be able to understand their clients' businesses and the markets in which those businesses operate. They also need to be able to understand, and articulate, the wider global context in which the products, services and systems they design will exist” (Design Council, 2010).

Here are the comments of 3 respondents on the choice of preferred competencies:

- $\quad$ "I never thought it could be that difficult. Although we talk, see each other, it also takes a long time to clarify all the details and understand some of the things in our work. Misunderstandings are our everyday life. I also think that business skills are especially important to us. In the online environment, however, it is even more challenging for us.” (Lucy, student of product design, on-line interview, June 28, 2020)

- $\quad$ "I perceive it positively. We will arrange everything you need online, and communication is simple. Definitely more than ever before." (Carl, student of product design, on-line interview, June 30, 2020)

- $\quad$ "It depends on the composition of the team and the communication we lead with each other. Being able to meet would be faster. From my point of view, all competencies are therefore important. And I perceive being able to understand clients as part of our daily work." (Lukas, professional product designer, on-line interview, June 25, 2020) 


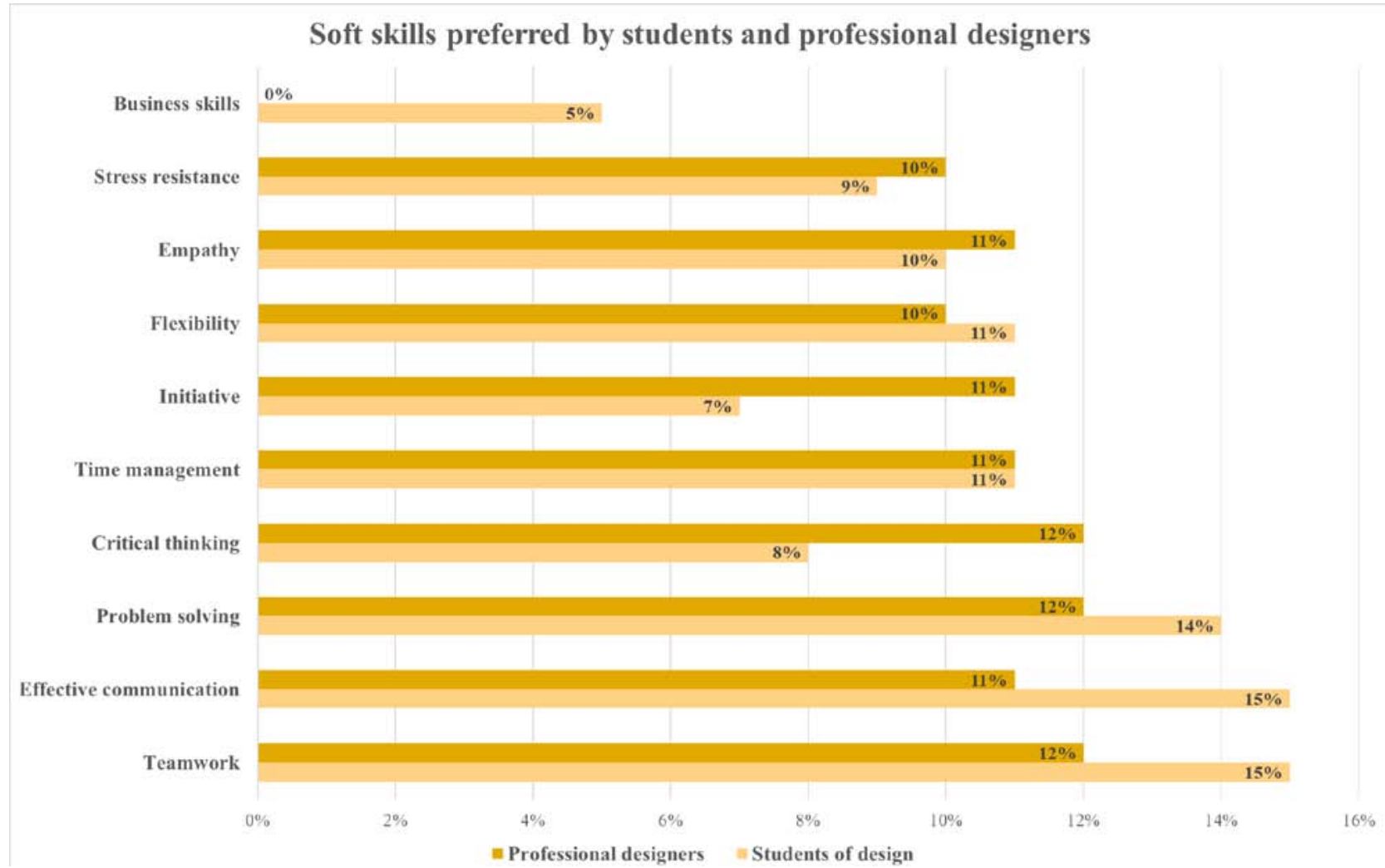

Figure 1. Results of international questionnaire survey of student and professional designers.

\section{Conclusion}

Soft competencies form one comprehensive group of skills. They are important for team interaction and also for managing own business. The international research de-scribed above shows that their importance in the digital age is no less than in the face-to-face time.

Tools for interdisciplinary creativity may vary, shift, and evolve with respect to digitization (McIntyre \& Watson, 2011), but some of the key skills of how to work in a team and be successful on the market seem to be preferred more than in common cooperation. Digitization has somewhat different demands, but it is necessary to work with this kind of environment, because it can establish new perspectives for relationship in the teams and between designers and their clients.

The results of research were quite surprising - there were identified the most preferred competencies as teamwork, problem solving, communication, time management and also business skills. This is also big opportunity for university's study plans, because they do not always offer courses in which young designers could develop their soft competencies. This creates an imbalance in the skills that designers have and need for their teamwork. It can stimulate a wider interest of students and motivate them to work in a team (Yanamandram \& Noble, 2006). Enabling students to acquire knowledge and experience in management and basic business principles is important for their future (Design Council, 2010).

\section{References}

Design Council. (2010). Multi-disciplinary design education in the UK. https://www.designcouncil.org.uk/sites/default/ files/asset/document/multi-disciplinary-design-education.pdf.

Findlay, R. (1997). The Bauhaus, and the collaborative critique. https://www.acsa-arch.org/chapter/gropius-the-bauhaus -and-the-collaborative-critique/.

Han, H.-Ch., Wright, J., Martinyuk, S., \& Ott, B. (2017). Art Education in the Era of Digital Visual Culture. The International Journal of Arts Education, 15(2), 79-90. 
Matteson, M. L., Anderson, L., \& Boyden, C. (2016). Soft skills: A phrase in search of meaning. Libraries and the Academy, 16(1), 71-88.

McIntyre, S., \& Watson, K. (2011). Online teamwork and collaboration. Learning to Teach Online, University New South Wales.

Meizlish, D., \& Anderson, O. (2018). Teaching in Teams: A Planning Guide for Successful Collaborations. http://crlt.umich.edu/sites/default/files/resource_files/CRLT_no37.pdf.

Nancarrow, S. A., Smith, T., Ariss, S., \& Enderby, P. M. (2014). Qualitative evaluation of the implementation of the Interdisciplinary Management Tool: a reflective tool to enhance interdisciplinary teamwork using Structured. https://www.researchgate.net/publication/269726573_Qualitative_evaluation_of_the_implementation_of_the_Interd iscipli-

nary_Management_Tool_A_reflective_tool_to_enhance_interdisciplinary_teamwork_using_Structured_Facilitated_ Action_Research_for_Implement.

Stewart A., \& Clifford R. (2018). New Models for Collaboration. In To get there: designing together (pp. 648-672). Paris.

Yanamandram, V., \& Noble, G. (2006). Student experiences and perceptions of team-teaching in a large undergraduate class. Journal of University Teaching and Learning Practice, 3(1).

Walker, K. (2018). A systems approach to design innovation. In C. Brunet (Ed.), To get there: designing together (pp. 674-696). Paris: E-book. 